Apidologie, 1979, 10 (3), 227-240.

\title{
QUANTITATIVE BESTIMMUNG DER 9-OXODECENSÄURE IM LEBENSZYKLUS DER KAPBIENE (APIS MELLIFERA CAPENSIS ESCHOLTZ)*
}

\section{Étude quantitative de l'acide céto-9 décène-2 ö̈que au cours du cycle biologique de l'abeille du Cap (Apis mellifera capensis Escholtz)}

\author{
C. HEMMLING, N. KOENIGER und F. RUTTNER \\ Fachbereich Biologie d.J.W.Goethe-Universität, \\ Institut für Bienenkunde \\ Im Rothkopf 5, D-6370 Oberursel/Ts.
}

\author{
SUMMARY \\ QUANTIFICATION OF 9-OXO-DECENOIC ACID \\ IN THE LIFE HISTORY OF THE CAPE HONEYBEE \\ (APIS MELLIFERA CAPENSIS ESCHOLTZ)
}

9-oxo-decenoic acid was quantified by a new, direct gaschromatographic analysis. Single newly emerged Cape honeybee workers were kept together each with 70-80 young workers of Apis mellifera car nica in small cages. The Cape bees started with oviposition on the 8th and 9th day under these conditions. 9-OD was found in $78 \%$ of all Cape workers in the experiments. The first occurrence of 9OD was noticed on the fourth day (after emergence) and the quantity of 9-OD increased with the age. On the 10th day an average of $50 \mu \mathrm{g} 9-\mathrm{OD}$ and on the 80 th day $300 \mu \mathrm{g} 9-\mathrm{OD}$ per worker were found.

Both, 9-OD production and oviposition were observed in 79 workers, while 8 worker bees had no 9 OD though they layed eggs. 12 out of 18 bees, which did not oviposit, produced 9-OD. So oviposition and 9-OD production are not directly correlated.

\section{ZUSAMMENFASSUNG}

Mit Hilfe einer neuen direkten gaschromatographischen Methode wurde die 9-Oxodecensäure (9-OD) quantitativ bestimmt.

\footnotetext{
* Mit Unterstützung der Deutschen Forschungsgemeinschaft.
} 


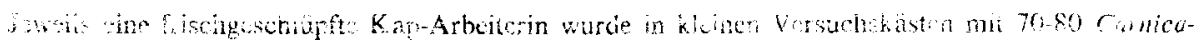

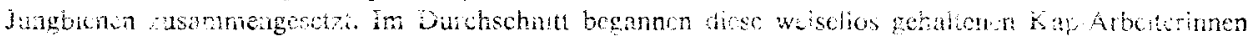

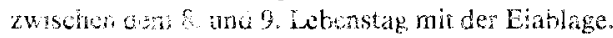

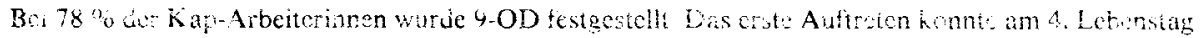

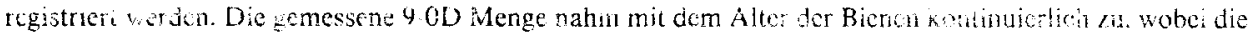
ermittelten Werts im unteren Bereich der Mengen lagen. dic be Koniginnen gunten wutun.

Sowohl Eiablage wie auch 9. OD Produktion wurde bei $74 \mathrm{~K}$ ap Arbeiterimun hohathtet. wahrend 8 cicilegende Arbeiterinnen keine 9 OD hatten. 12 von 18 Bienen, die keine Eler iegten, produziorten 9-9D. Eilage und $y$ OD Produktion hängen demnach nicht direkt voneinander $a b$.

\section{ERLETUNG UND PROBI FMSTELLUNG}

Als Hauplkompuiente des Mandibeldrüsensekrets der Königin wurdo tian,-9Oxo-2-decensäure (9-OD) isoliert (BAREIER und Lererer, 1960; CALlow und JOHNSTON, 1960).

RUTRER el al. (1976) konnien 9 OD erstmals auch bei legenden Arbeiterinnen von Apis mellifera cafensis nachwessen.

Dicser erste nur wenige Proben umfassende achwes von 9 OD hei Arbeitsbie nen war die Grundlage für diese Untersuchung, die sich mit tolgenden Fragestellungen befasste :

1. Ist die Bildung yon 9 OD eine Eigenschaft aller Kap Arbeiterinnen, oder ist sie auf legende Arbeitetinnen beschränkt?

Ist die Ovarentwicklung mit der 9-OD-Produktion gekoppelt?

2. In welchen Alter beginnt die G OD Bildung, und wie verändert sich die Pheromonmenge in Abhängigkeit vom Lebensalter der Kap-Arbeiterinnen?

3. Sind die bei den Kap-Arbeiterinnen gefundenen Ergebnisse mit denen von Königinnen zu vergleichen?

\section{MATEKIAL UND METHODE}

\section{Material}

Für die Versuche wurden Arbeiterinnen von Apis mellijera capensis ESCHOLTZ verwendet, einer Rasse, die seit einigen Jahren am Institui für Bienenkunde in Oberursel gehalten wird.

Das natüriiche Verbreitungsgebiet der Kapbiene ist die Südspitze Afrikas im eng begrenzten Gebiet der Kapregion. Von anderen weiter nördlichen Bienenrassen (scutellata-adansonit-Gruppe) kann die Capensis mit den üblichen biometrischen Methoden nicht einwandfrei getrennt werden, da äussere Merkmale, wie dunkle Körperpigmentierung und eine erhöhte Borstenzahl auf den Vorderflügeln, keine ausreichenden Unterscheidungsmerkmale darstellen (RUTTNER, 1977).

Die exakten Kriterien sind in der Art der Reproduktion und in der Ausbildung der Fortpllanzungsorgane bei den Arbeiterinnen zu finden. Aus den unbefruchteten Eicru von Kap Arbeiterinnen entwickeln 
sich normale weibliche Tiere -..- thelytoke Parthenogenese (ONIONS, 1912; RUTNER, 1976) -.., während aus den Eiern von Arbeiterinnen anderer Rassen Drohnen schlüpfen.

Daneben sind die Arbeitsbienen von A.m. capensis schon von ihrer Anlage her königinnenähnlicher als Arbeiterinnen anderer Rassen. Sie besitzen eine Spermatheka und eine erhöhte Ovariolenzah.

\section{Methode}

\subsection{Erzeugung und Haltung von legenden Kap-Arbeiterinnen}

Die für die Versuche notwendige grosse Anzahl von legenden Kap Arbeiterinnen definierten Alters wurde auf folgende Weise erreicht.

In kleine Holzkäfige $(7,5 \times 7 \times 9 \mathrm{~cm})$ wurde je eine frischgeschlüpfte, markierte Kap-Arbeiterin mit 70-80 Jungbienen von A.m. carnica zusammengesetzt. Dieser Ansatz beruht auf der unterschiedlichen Entwicklungszeit der Ovarien bei den genannten Rassen. Die Kap-Arbeiterinnen benötigen eine wesentlich kürzere Zeit bis zur Fntwicking ihrer Ovarien (6-7 Tage) als die Carnica-Arbeiterinnen (etwa 30 Tage: HESSE, 1977).

Die Bienen wurden über die gesamte Versuchsdauer mit Zuckerteig, Wasser und frischem Pollen versorgt. In jedem Versuchskäfig war ein kleines Stück bereits bebaute Mittelwand (ca. $5 \times 3 \mathrm{~cm}$ ) angebracht (PAIN, 1966). Täglich erfolgte eire Inspektion der Kästen mit Fütterung, Feststellung einer etwaigen Eiablage sowie Entfernung der toten Bienen. Die Versuchskästen wurden im Brutschrank bei $27^{\circ} \mathrm{C}$ gehalten. Nach 30-35 Tagen Versuchsdauer stieg der Totenfall der Camica-Bienen meist so stark an, dass es notwendig war, die Kap-Arbeiterin in ein neues Kästchen mit Jungbienen umzusetzen. Während der 1. Periode (2.-12. Tag) wurden für jeden Tag 10-20 Versuchskästen angesetzt. Vom 15.-50. Lebenstag wurde ein 5-Tage Rhythmus eingehalren, mit 5-10 Kästchen pro Ansatz. Insgesamt wurden $246 \mathrm{Kap}$-Arbeiterinnen bekannten Alters ausgewertet, gewonnen aus einem Ansatz von ursprünglich $254 \mathrm{Kästchen.} \mathrm{Die} \mathrm{Altersver-}$ teilung der Arbeiterinnen zur Zeit der Auswertung ist aus Tab. 1 ersichtlich. Die Bienen wurden bis zur weiteren Verarbeitung bei ca. $-16^{\circ} \mathrm{C}$ aufbewahrt.

Als Kontrollversuch wurden zur selben Zeit Kap-Aibeiterinnen einem weiselrichtigen Kap-Volk zugesetzt. Die im Brutschrank bei $32^{\circ} \mathrm{C}$ geschlüpften Kapbienen wurden täglich von der Brutwabe abgesammelt und vor dem Zusetzen in das Kapvolk farbig markiert. Insgesamt wurden dem Kapvolk von Mitte Juli bis Ende August 1200 gezeichnete Arbeiterinnen zugegeben. Vom 2.-10. Lebenstag der KapArbeiterinnen wurden täglich 1020 Bienen abgeingen. Vom 15 bis 60 . Lebenstag wurden die $\mathrm{Kap}$ Arbeiterinnen im 5-Tage-Rhythmus abgesammeit.

\section{Präparation der Kap-Arbeiterinnen}

Die Präparation der Ovarien erfolgte nach der Methode von G. Hess (1942). Bei 20-facher Vergrösserung wurden die Ovarien unter dem Binokular ausgewertet.

Als Kriterium für die Beurteilung des Entwicklungsgrades der Ovarien wurde die Kammerung der Ovariolen gewählt. Es erfolgte eine Einteilung in 3 Stufen (SAKAGAM und AKAHRA, 1958; VkLtrus, 1970) :

Mit Stufe 1 wurden alle Ovarien bezeichnet, die völig unentwickelt waren, d.h. bei denen die Ovario len keine Einschnürungen aufwiesen.

Unter Stufe 3 wurden die Ovarien gezählt, bei denen legereife Eier in den Ovariolen erkennbar waren.

Stufe 2 umfasste alle Zvischenformen, d.h, vom Beginn der Kammerung der Ovariolen bis hin zu ganz deutlicher Kammerung.

Die Spermatheka, die an der Stelle liegt, wo sich der Oviductus communis in die beiden lateralen Ovidukte aufspaltet, wurde bei 40 facher Vergrösserung unter dem Binokular ausgemessen. Bei jeder Sperma. theka wurden zwei senkrecht aufeinanderstehende Durchmesser angegeben.

\subsection{Quantifzierung der 9-Oxodecensäure}

Der klassische Nachweis von 9OD (CALLOW und JoHNs roN, 1960) wird über eine Veresterung durchefubrt. Dabei werden die Kogle her za untersuchenden Bienen mit Ätser extrahiert und die Säu- 
ren durch Zugabe von Diazomethan in die entsprechenden Methylester überführt. Erst dann kann die gaschromatographische Aufarbeitung erfolgen. Um die quantitativen Ungenauigkeiten. die bei der Methylierung mit Diazomethan entstehen, auszuschliessen und um die Empfindlichkeit auf die für diese Untersuchung erforderliche Stufe zu erhöhen, haben wir direkt chromatographiert. Diese Messmethode hat neben geringerem Arbeitsaufwand den Vorteil, dass auch sehr kleine Mengen 9-OD gemessen werden können. Die Nachweisgrenze liegt bei ca. $0,5 \mu \mathrm{g}$.

Die Messungen erfolgten an einem Gaschromatographen der Firma Hewlett-Packard Series 57201. Es wurde mit einem FID und einer Stahlsäule (Länge $1,8 \mathrm{~m}$, Durchmesser aussen 3,2 mm, innen 2,7 mm, 2,5\% SP 1000, Chromosorb G, AW-DMCS, 80-100 mesh) gearbeitet. Die Temperatur im Säulenofen war konstant $240^{\circ} \mathrm{C}$ (Einspritzblock $280^{\circ} \mathrm{C}$, Detektor $300^{\circ} \mathrm{C}$ ).

Als Trägergas wurde Stickstoff verwendet (Strömungsgeschwindigkeit $40 \mathrm{ml} / \mathrm{min}$ ).

Zur Messung wurde der Kopf einer einzelnen Kap-Arbeiterin in einem Eppendorffhütchen mit 50 . $100 \mu 1$ Aceton als Lösungsmittel zerquetscht. Nach 15-stündiger Aufbewahrung im Kühlschrank erfolgte die gaschromatographische Messung, wobei 1-3 $\mu$ des Acetonextrakts in den Probeneinlass gegeben wurden. Wenn mehrere Köpfe von gleichaltrigen Arbeiterinnen zusammen in einer Probe verarbeitet wurden, erhöhte sich die Menge des Lösungsmittels entsprechend.

Bei den Kapbienen aus dem weiselrichtigen Volk wurden für jede Altersstufe 10 Bienen untersucht, wobei 2 Messungen mit jeweils 5 gleichaltrigen Bienen erfolgten. Bei den Kap-Arbeiterinnen aus den Versuchskästen wurde dagegen grösstenteils Einzelmessungen vorgenommen. Am 2., 3., 5., 6., 7., 9. und 11 . Lebenstag wurden allerdings 2-6 Köpfe jeweils in einer Probe aufgearbeitet.

TAB. 1. - Altersverteilung der Kap-Arbeiterinnen zum Zeitpunkt der Auswertung.

TABL. 1. - Distribution de l'âge des ouvrières du Cap au moment de l'expérience.

\begin{tabular}{|c|c|c|c|c|c|c|c|c|c|}
\hline $\begin{array}{l}\text { Alter der Kap- } \zeta \bar{\gamma} \text { in Tagen } \\
\text { Age en jours des } Y \zeta d u \text { Cap }\end{array}$ & $2 \cdot 5$ & $6-9$ & $10-15$ & $16-20$ & $21-30$ & $31-40$ & $41-50$ & $51-75$ & $>80$ \\
\hline $\begin{array}{l}\text { Anzahl der Kap- } \nvdash \zeta \\
\text { Nombre d' } \zeta \zeta \text { du Cap }\end{array}$ & 41 & 62 & 37 & 20 & 18 & 31 & 16 & 10 & 11 \\
\hline
\end{tabular}

Mit Hilfe einer Vergleichslösung aus synthetischem 9. OD, in Aceton gelöst, die nach jedem Chromatogramm mit Kopfextrakt gespritzt wurde, konnte ermittelt werden, ob in der untersuchten Probe 9-OD vorhanden war und wenn ja, in welcher Menge (Abb. 1).

Die quantitative Auswertung erfolgte über eine angenäherte Flächenbestimmung des Peaks : Peakhöhe $\times$ Halbwertsbreite. (Halbwertsbreite $=$ Breite eines Peaks in halber Höhe zwischen Maximum und Grundlinie.)

\section{ERGEBNISSE}

\section{Verhalten der Bienen in den Versuchskästen}

\section{Bautätigkeit}

Bei der täglich durchführten Inspektion wurde für jeden Versuchskasten der Beginn des Zellenanbaus an das eingeklebte Wabenstück bestimmt, wobei als Kriterium deutlicher Anbau in Form von mindestens einer Zelle gewählt wurde. 


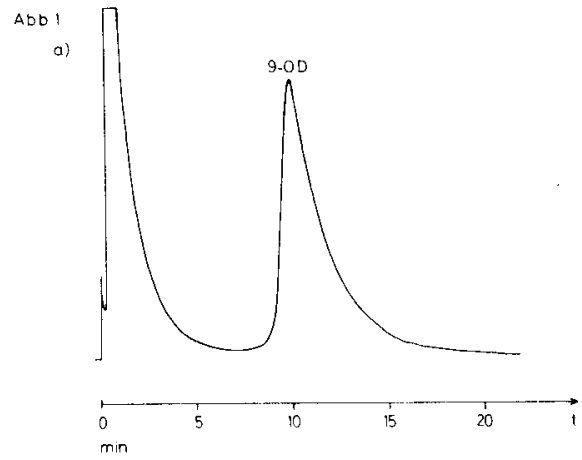

b)
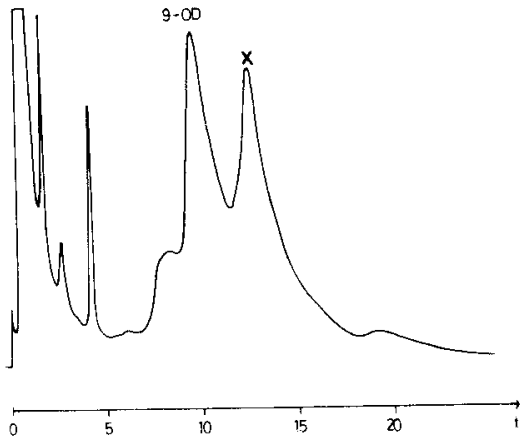

min

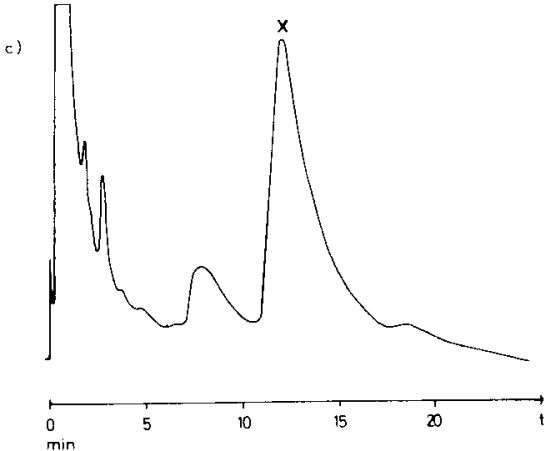

Aвв. 1. - Gaschromatogramme.

a) $4 \mu \mathrm{g}$ synthetische 9-OD

b) $2 \mu$ l Extrakt (5 20-tägige Kap- $\nvdash$ aus dem weiselrichtigen Volk, in $150 \mu 1$ Aceton gelöst) $\mathrm{X}=$ nicht identifizierter Peak (9-Hydroxy-Decensäure?)

c) $1 \mu \mathrm{\gamma}$ Extrakt (45-tägig, in $100 \mu 1$ Aceton gelöst), $276 \mu \mathrm{g}$ 9-OD $\mathrm{X}=$ nicht identifizierter Peak (9-Hydroxy-Decensäure?)

FIG. 1. - Chromatogrammes gazeux

a) $4 \mu \mathrm{g}$ d'acide céto-9 synthétique

b) $2 \mu 1$ d'extrait de 5 ouvrières du Cap âgées de 20 jours, provenant d'une colonie avec reine et macérées dans $150 \mu 1$ d'acétone

$\mathrm{X}=$ pic non identifié (acide hydroxy-9 décen-2 oïque?)

c) $1 \mu 1$ d'extrait d'ouvrière (âgée de 45 jours, dissout dans $100 \mu 1$ d'acétone), $276 \mu \mathrm{g}$ d'acide céto 9 . 
Zwischen dem 5. und 7. Versuchstag war die grösste Bauaktivität zu beobachten. Bis einschliesslich des 6. Versuchstages hatten die Bienen in 39,9\% der Kästen mit dem Anbau von Zellen an das Wabenstück begonnen. Am Ende des 7. Versuchstages wie sen schon $60,1 \%$ der Kästen angebaute Zellen auf. Wenn bis zum 10. Versuchstag keine Zellen angebaut wurden, blieb das Wabenstück bis zum Versuchsende unberührt. Das war bei $22,3 \%$ der Kästen der Fall.

Für jede Kap-Arbeiterin wurde der Beginn der Eiablage bestimmt (Tab. 2). Im Durchschnitt begannen die Kap-Arbeiterinnen am 8,7. Lebenstag mit der Eiablage. Wenn die Arbeiterinnen bis zum 15. Lebenstag nicht legten, gingen sie auch später nicht mehr in Eilage, Von 105 Arbeiterinnen, die ein Alter von mehr als 15 Lebensta gen erreichten, legten $18(=17,1 \%)$ während der gesamten Versuchsdauer keine Eier.

Bei der täglichen Inspektion der Versuchskästen wurde in vielen Fällen ein Hofstaat um Kap Arbeiterinnen beobachtet. Diese mehr zufälligen Beobachtungen erlaubten keine systematische Auswerturg.

TAB 2. - Beginn der Eilage von $138 \%$ Y̧

TAuL 2. - Début de la ponte chez 138 ouvières.

\begin{tabular}{|c|c|c|c|c|c|c|c|c|c|c|}
\hline $\begin{array}{l}\text { Tag } \\
\text { Jour }\end{array}$ & 5. & 6. & 7. & 8. & 9. & 10 & 11. & 12. & 13. & 14. \\
\hline 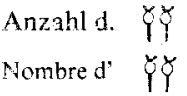 & 1 & 1 & 20 & 50 & 38 & 13 & 8 & 4 & 1 & 2 \\
\hline
\end{tabular}

\section{Alter der Kap-Arbeiterinnen}

Über das Lebensalter und die Dauer der Eilage kann nur bei einigen KapArbeiterinnen eine Aussage gemacht werden, da die Versuche vom Ansatz her ein Abtöten der Bienen nach bestimmten Zeitintervallen bedingten. $13 \mathrm{Kap}$-Arbeiterinnen erreichen eine Lebensdauer von über 75 Tagen. 4 Arbeiterinnen legten im Alter von über 100 Tagen $(102,103-, 116$ - und 126-tägig) noch Eier. Die älteste K.ap-Arbeiterin wurde 137 tägig getötet.

\section{Spermatheka und Ovarentwicklung der Kap-Arbeiterinnen}

Von 190 präparierten Kap Arbeiterinnen aus dem weiselrichtigen Volk wurde hei $66 \%$ eine Spermatheka gefunden. Die Grösse betrug im Durchschnitt $0,45 \times 0,44 \mathrm{~mm}$ (min : $0,27 \times 0,27 \mathrm{~mm}$; max. : $0.55 \times 0,52 \mathrm{~mm}$ ) und war vom Alter der Bienen unabhängig.

In den Versurpskästen wiesen von 221 untersuchten Kap Arbeiterinnen 64,7\% eine Soematheka aut Die durchechnittliche Grösse betrug $0,5 \times 0,5 \mathrm{~mm}$ (min: 


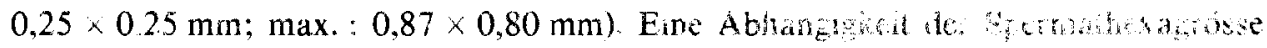
vom Alter der Bienen konnte auch hier nicht iestgesabli buder.

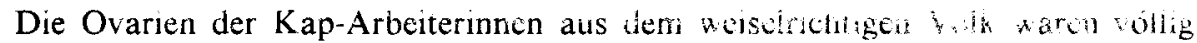
unentwickelt, d.h. sie wurden der Entwicklungsstufe 1 zugeridne:

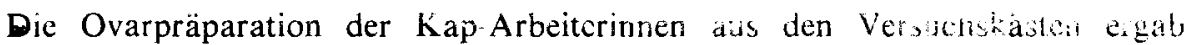
folgende Ergebnisse: Am 2. und 3. Lebenstag waden nur anenrivickite O arien gefunden. Am 4. Lebenstag konnien bereits bel ener hap Arbuterin Orantin mit Entwicklungsstufe 2 registriert werden. Am 5. Tag waren es voi 12 Bienen schon 5 , die entwickelte Ovarien aufwiesen

\section{9.OD Menge in den Kap Albeiterimen}

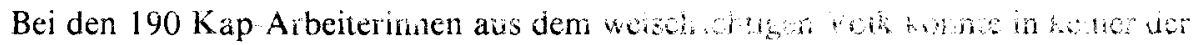
38 untersuchten Bienenproben 9 OD nachgewiesen wisten

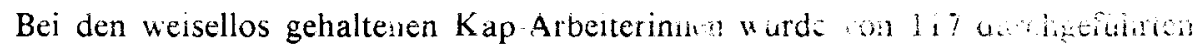
Einzelmessungen bei $78 \%$ der Arbeiterinnen $9.0 \mathrm{O}$ festgest ilt.

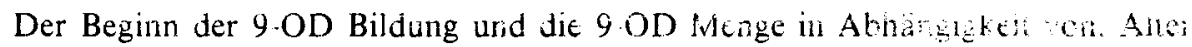
der Kap-Arbeiterinnen wird an der Kurve in Abb. 2 deutlicn.

Der Verlauf der Kurve zeigt, dass die gemessen 9-OL Menzo wit do Altor der Kap-Arbeiterinnen hontiouierlich zummmt (Regresslonsgerade: lit - 3.18; $\mathrm{y}(\mathrm{x}=0)=24,22$ ). Das erste Auftreten von 9-01 wurde am 4. L.abinsias der Kap Arbeiterinnen registriert. Von 10 einzeln intersichien Kap Aibeiterimen diser Altersstufe konnte bei 4 Tieren 9-OD festgestelit werden. Dic durchschnittiche Menge betrug 12,5 $\mu \mathrm{g}$ pro Arbeiterin. Der höchste 9-OD Wort aller Einzelmesstingen wurde bei einer 111 Tage alten Kapbiene mit 633,6 $/ \mathrm{g}$ gemessen.

\section{9-OD Menge und Oiarentwickising}

Wird der 9-OD Gehalt in Zusammenhang mit der Ovarentwicklung de: Kap Arbeiterinnen betrachtet, so zeigen sich folgende Ergebnisse : Gleichzeiitg mii den ersten Auftreten von entwickelten Ovarien am 4. Lebenstag der Kap-Arbeiterinnen wurde, wie schon oben angeführt, zum ersten Mal 9-OD fesigestellt.

Parallel zur Häufigkeit von Arbeitsbienen mit entwickelten Ovarien erhöht sich auch die 9-OD-Menge. Diese Parallelentwicklung gilt für Arbeiterinnen bis zum 10. Lebenstag, später jedoch nicht mehr. Während sich dann die mittlere Ovarstufe der älteren Arbeiterinnen (vom 10. bis 50. Lebenstag) auf einen Wert um 3 einstellt (d.h. fast alle Kap-Bienen legen Eier), nimmt die gemessene 9-OD-Menge noch weiter zu. Bei Kap-Arbeiterinnen mit einem Alter von mehr als 50 Tagen, bei denen zum Zeitpunkt der Messung die höchsten 9-OD-Werte erhalten wurden, waren die Ovarien zwar immer noch sehr stark entwickelt, legereife Eier wurden dagegen nur selten gefunden. Der Entwicklungsgrad wurde daher mit Stufe 2 bewertet. 


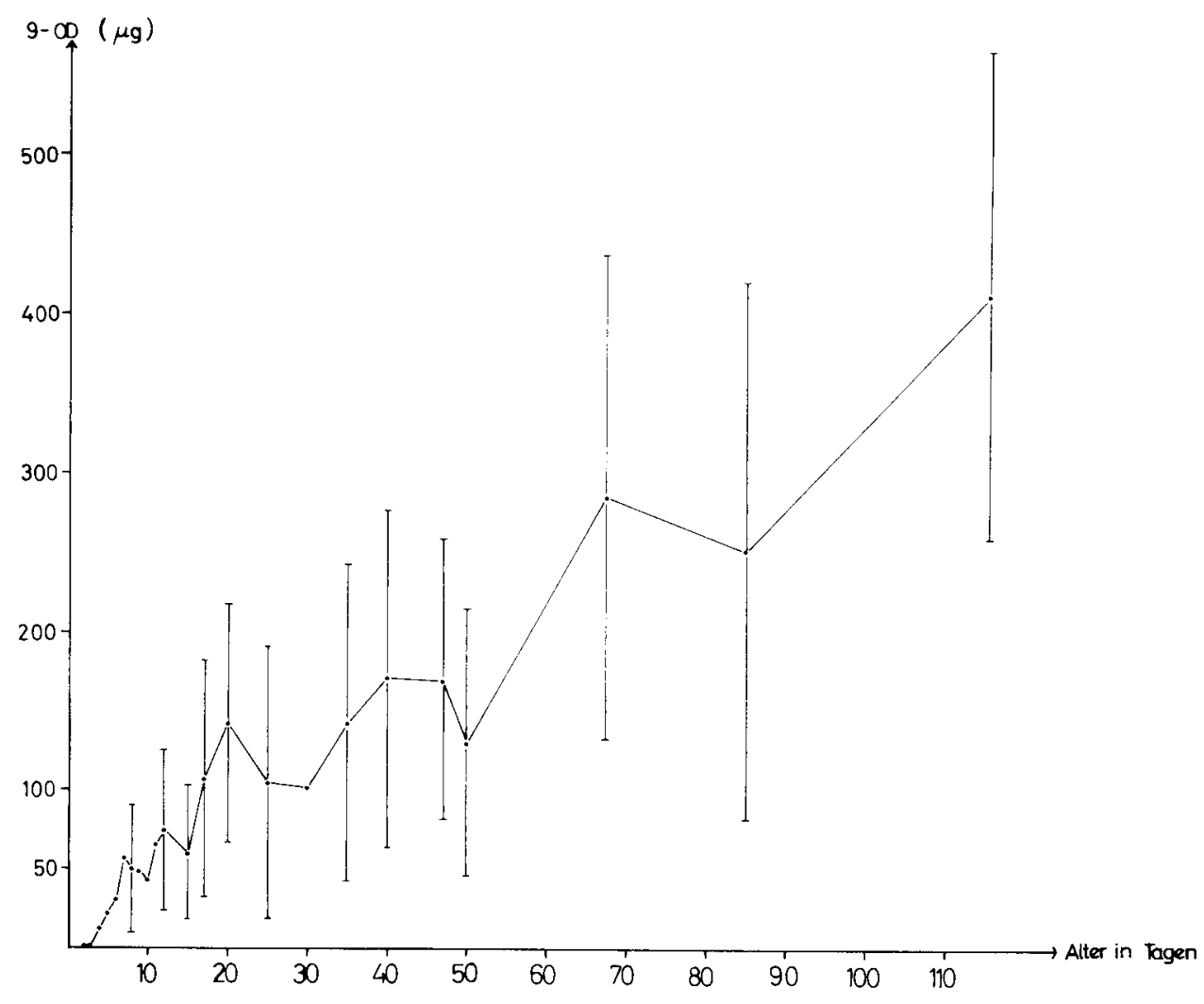

Авв. 2. - 9-OD Menge in Abhängigkeit vom Alter der $\zeta \zeta$

Abszisse : Alter der $Y \gamma$ in Tagen

Ordinate : 9-OD $(\mu \mathrm{g})$

Fig. 2. - Quantité d'acide céto-9 en relation avec l'âge des ouvrières

Abscisses : âge des ouvrières en jours

Ordonnées : acide céto-8 $(\mu \mathrm{g})$

Die Tatsache, dass keine legereifen Eier in den Ovarien zu finden waren, bedeutet aber noch nicht, dass die Kap-Arbeiterinnen die Eilage völlig beendet hatten und nicht mehr in der Lage waren, erneut Eier zu legen. Das Alter der Begleitbienen hat nach unseren Beobachtungen einen entscheidenden Einfluss auf die Legetätigkeit der KapArbeiterinnen. Ein Beleg für diese Annahme ist darin zu sehen, dass 15 von $22 \mathrm{Kap}-$ Arbeiterinnen durch Umsetzen in Kästen mit Carnica-Jungbienen ihre unterbrochene Legetätigkeit wieder aufnahmen.

Kap-Arbeiterinnen, die in den Versuchskästen bis einschliesslich des 15. Lebenstages nicht mit der Eilage begonnen hatten, legten bis zum Versuchsende keine Eier mehr. Von 18 Kap-Arbeiterinnen, die älter als 15 Tage waren und keine Eier legten, wurden bei 10 Tieren Ovarien mit Entwicklungsstufe 2 und bei 8 Tieren Ovarien 
mit Entwicklungsstufe 1 gefunden. Über den Zusammenhang zwischen Eilage und 9-OD-Gehalt gibt Tab. 3 Aufschluss. Der Anteil der Tiere, die 9-OD produzieren, ist bei den eierlegenden Arbeiterinnen signifikant grösser $\left(x^{2}=7,45\right)$ als bei den Bienen, die während der Versuche keine Eier legten. Betrachtet man jedoch bei beiden Gruppen nur die Tiere mit positivem 9-OD-Befund, so ergibt sich kein signifikanter Unterschied in der Menge (151,6 $\mu \mathrm{g}$ bei Eilage und 194,3 $\mu \mathrm{g}$ ohne Eilage).

TAB. 3. - 9-OD Produktion und Eilage

(Arbeiterinnen älter als 15 Tage).

TABl. 3. - Production d'acide céto-9 et ponte

(ouvrières âgées de plus de 15 jours).

\begin{tabular}{l|c|c}
\hline \hline & $\begin{array}{c}\text { 9-OD } \\
\text { Acide céto-9 }\end{array}$ & $\begin{array}{c}\text { Keine 9-OD } \\
\text { Pas dacide céto-9 }\end{array}$ \\
\hline $\begin{array}{l}\text { In Eilage } \\
\text { Ponte }\end{array}$ & 79 & 8 \\
\hline $\begin{array}{l}\text { Nicht in Eilage } \\
\text { Pas de ponte }\end{array}$ & 12 & 6 \\
\hline \hline
\end{tabular}

\section{DISKUSSION}

\section{Verhalten der Bienen in den Versuchskästen}

\subsection{Bautätigkeit}

Die Beobachtung, dass die Begleitbienen zwischen dem 5. und 7. Versuchstag mit dem Anbau von Zellen an das eingeklebte Wabenstück begannen, stimmt mit den Angaben von ROGER (1971) überein. In Versuchskäfigen mit 50 Bienen konnte er den Anbau von Zellen an die Mittelwand zwischen dem 6. und 7. Lebenstag feststellen, wobei er der Königin eine primäre Bedeutung für das Bauverhalten zuschreibt. DARCHEN $(1957,1960)$ hatte zuvor in Versuchen mit unterschiedlicher Bienenzahl diesen Einfluss der Königin genauer beschrieben. Bei Anwesenheit einer Königin, unbegattet oder begattet, konnte auch er bei 50 sechstägigen Arbeiterinnen eine Bautätigkeit feststellen. Ohne Königin wurde nur gebaut, wenn 10000 Bienen vorhanden waren. Bei Anwesenheit von eierlegenden Arbeiterinnen genügten nach den Versuchen von DARCHEN bereits 5000 Bienen, um ein Bauverhalten auszulösen.

Unter unseren Versuchsbedingungen haben sich die Begleitbienen so verhalten, wie in den Versuchen von DaRCHEN bei Anwesenheit von einer Königin. So kann die Beobachtung der Bautätigkeit in den Versuchskästen bereits einen ersten Hinweis 
darauf gehen, dass die Kap A.hettrinnen anf die Begleitbienen eine Wirkung als "Ersatrlanigirara n auribet.

\section{Eirblase}

Es ist bekannt. dass sich bei Weisellosigkeit die Ovarien der Arbeitsbienen encicksm, und legende Arteterinner aufteter: (DF Groor and Voogd. 1954; VuOU, 1955. 1956; BUTLE: 1957 b).

Zwischen sen einzelnen Rassen bestehen jedoch Unterschiede in der Latenzzeit, d.h. in der Zeit, die die Ovarien benötigen, um sich zu entrickeln, und die ersten Ejer abgelegt werds rönen.

Versuche an reinen Kap-Völkern ergaben, dass nach Entfernen der Königin die ersten Eier noch schneller aultreten als in den Versuchskästen, in denen Carnica Jurgbienen als Begleitbienen verwendet wurden : nach 2-3 Tagen (ONIONs, 1912), nach 4.8 Tagen (A IDFesom, 1963) und nach 6-7 Tagen (Hesse, 1977)

Das Vorhandensein von Eiern am 2. bis 3. Tag nach Entweiselung kann wohl nur damit erklärt werden, dass immer enige Arbeiterinnen mit bereits entwickelten Ovarien (Entxicklungsstufe 2.3) in Volk anwesend sind. Diese Annahme wird von der Beobachtung A DERsows (1963) bestätigt, der in fast allen Kolonien trotz

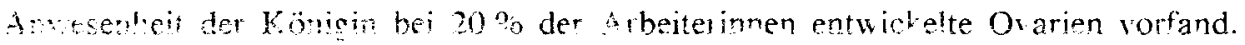

\section{Alto der Kap Arbeiterinne!}

Über die Lebensdauet vou legenden Kap-Arbeiterinnen liegen noch keine Untersu chungen $\mathrm{V}$, aber unsere Ergebnisse zeigen, dass legende Arbeiterinnen in Versuchskästen bis 20140 Tager alt werden können. Sommerbienen in frei fliegenden Ver suchsvölkerr haben eine Lebensdauer von 25-35 Tagen, max.60-70 Tagen, während Winterbienen 6.7 Monate alt werden können (MACRIzIO, 1946, 1961). BerTHOLF (1942) konnte zeigen, dass weder die Arwesenheit einer Königin, noch die Möglichkeit, Reinigungstlüge $z$ u lutermehrea, tiven Finfluss auf die Lebensdauer von gefangen gehaltenen Bieren hat.

Neben zahlreichen morphologischen Verschiedenheiten zwischen adulten Königinnen und Aibeiterinner besteht ein wesentlicher Unterschied beider Kasten in der Lebensdauer. Durch tie gegenüker reproduktiv inaktiven Arbeiterinnen verlängerte Lehersait ist die logende $K a p$ arbetsbiete auch in diesem Nerkmal königinnenähnli cher.

\section{Spernatheka bei Kap-4rbziterinnen}

Die Grösse der Spermatheka liegt mit einem Durchmesser von durchschnittlich $0,45 \times 0,44 \mathrm{~mm}$ im weiselrichtigen Volk und $0,5 \times 0,5 \mathrm{~mm}$ in den Versuchskästen im Bereich der Werte we se fir an Arbeiterinnen bereits beschrieben wurden (PUT NFR, 1977) 
ANDERSON (1963) stellte eine geringe Grossenzunahme der Spenthathek bei legenden Arbeiterinnen im Vergleich zu nicht legenden fest : Spomatheka bei legenden Arbeiterinnen 0,29 $\times 0,35 \mathrm{~mm}$, bei nicht legenden $0,25 \times 0,3 \mathrm{~mm}$. Grössenunterschiede in der gleichen Ordnung, von 0,04 $0,06 \mathrm{~mm}$, konnten auch bei unseren Messungen beobachtet werden.

Der Nachweis ciner Spermatheka bel $65 \%$ der Versuchsbiench ist zugleich ein Beweis dafür, dass es sich tatsächlich um Kap-Arbetterinnen gehandeit hat. CarnicaArbeiterinnen besitzen höchstens Rudimente von Spermatheken.

\section{9.OD Menge in den Kap-Arbeiterianen}

Da bis jetzt noch keine quantitativen Untersuchugen über den 9 - OD Gehalt hei legenden Arbeiterinnen vorliegen, werden die bei Kap-Arbeiterinnen trhaitenen Ergebnisse mit solchen von Königinnen verglichen.

Bei Königinnen wurde bereits am ersten Tag nach dem Schlüpfen 900 festge

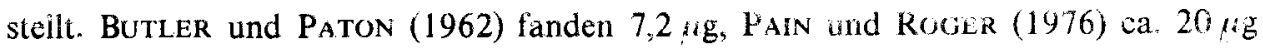
9-OD pro Königin. Bei Kap-Arbeiterinnen konnte das erste Auftreten yon 90 O erst am 4. Lebenstag mit einer durchschnittlichen Menge son 12,5, $\mathrm{g}$ pro Arbeiterin registriert werden. Die 9.OD Menge nimmt dann mit dem Alter kontinuierlich za. Ob die hohen Werte an 9 Oxodecensäure bei alten Arbeiterinnen auf eine vermehrta Bildung des Sekrets oder auf eine Akkumulation zurückgehen, bleibt zu diskutieren. Möglich wäre, dass ältere Begleitbienen aufgrund eines reduzierten Futieraustausches die legende Arbeiterin nicht mehr so oft berühren, und sich dadurch die Menge an 9. OD in dieser anreichert. Die mit dem Alter der Kap-Arbeiterinnen registrierte Zunahme des 9Oxodecensäure-Gehalts muss deshalb nicht unbedingt auf eine vermehrte 9-OD Bildung zurückgehen, denn es kann auch so sein, dass stäidig mehr 9.OD produziert als abgegeben wird.

Die kontinuterliche Zunahme der 9 Oxodoensäure mit den Altor der KapArbeiterinnen entspricht den Angaben, wie sie von BUTLR und PATON (1962) ind PAIN et al. $(1962,1967)$ für Königinnen gemacht wiurden.

PAIN und ROGER (1976), die ebenfalls die 9. OD Menge in Ariangigheit rom Alter unbegatteter Königinnen untersuchten, steilten jedoch ein 9.OD Maximum bei 16-20-tägigen Königinnen fest (durchschnitilich / $107 / \mathrm{g}$ ). Mit zunchmendem Alter der Königinnen sank die 9-OD Menge dann wieder ab (bei 46 bis 50 ) tägigen auf $282 \mu \mathrm{g})$. Eine Erklärung für die verminderte 9-OD Merige im Alter wird von den Autoren in den unnatürlichen Versuchsbedingungen gesehen. Sie netmen an, dass ältere Begleitbienen der Königin nicht mehr alle wichtıgen Elemente liefern, die sie benötigt, um 9-OD zu bilden. Demnach ist die 9-OD Menge stark abhängig von physiologischen Zustand, dem Alter und der Anzahl der Begleitbienen (PAls et al, 1972).

Ein Vergleich der 9-OD Miengen zwischen Kap-Äbeiterinnen ind Könininnen zeigt, dass die durchschnithlichen Werte, die bei legenden Kap Arbeiterimen gefinden 
wurden, durchaus mit denen von Königinnen zu vergleichen sind, obgleich die bei diesen gefundenen Höchstwerte in unserer Versuchsserie nicht erreicht wurden.

Grosse individuelle Unterschiede im 9-OD Gehalt, wie sie für gleichaltrige KapArbeiterinnen bei dieser Untersuchung gefunden wurden, sind auch bei Königinnen gleichen Alters bekannt (PAIN et al., 1967).

Ovarentwicklung bzw. Eilage und 9-OD-Produktion treten bei den meisten hier untersuchten Bienen gleichzeitig auf. Aber auch Bienen, die nicht in Eilage gegangen sind, zeigen 9-OD-Produktion. So kann vermutet werden, dass in der Regel beide Organe (Mandibeldrüse und Ovarien) bei Weisellosigkeit über neurosekretorische Zentren aktiviert werden. Jedoch scheint wie bei der Königin (BUTLER, 1959; GARY, 1961) keine direkte Verknüpfung zu bestehen.

Eingegangen im April 1979. Reçu pour publication en arril 1979.

\section{RÉSUMÉ}

Il existe de nombreuses données concernant la corrélation entre l'âge et la production d'acide céto- 9 décène-2 oïque (acide céto-9) chez les reines (ButLer et Paton, 1962; PAin et Roger, 1976, 1978; Pain et al., 1967, 1972, 1974). Dans ce travail on a cherché à savoir si la quantité de phéromone variat également avec l'âge chez les ouvrières pondeuses.

La détermination quantitative de la teneur en acide céto-9 en fonction de l'àge a été effectuée chez les ouvrières d'A.m. capensis. Les ouvrières des abeilles du Cap (Origine : pointe sud de l'Afrique) se distinguent par une caractéristique biologique : les œufs des ouvrières pondeuses donnent naissance à des individus femelles diploïdes (parthénogenèse thélytoque). En outre les ouvrières du Cap sont de prime abord plus proches d'une reine que des ouvrières des autres races : elles possèdent une spermathèque (diamètre 0,3 $0,8 \mathrm{~mm})$ et un nombre élevé d'ovarioles (15-30).

Dans des cagettes expérimentales on a rassemblé $70-80$ jeunes abeilles d'A pis mellifica carnica sans reine et une ouvrière du Cap fraichement éclose et marquée. Cette disposition garantit que les œufs qui vont apparaitre les premiers proviennent du Cap.

Les cagettes étaient munies d'un petit morceau de rayon et conservées à l'étuve à $27^{\circ} \mathrm{C}$. Les abeilles étaient approvisionnées en eau, candi et pollen. Comme témoin on a ajouté des ouvrières du Cap fraîchement écloses et marquées à une colonie du Cap possédant une reine.

On a tué à intervalles déterminés les ouvrières du Cap des cagettes expérimentales et celles de la colonie avec reine. On a examiné l'état de développement de leur ovaires, mesuré la spermathèque, quand elle existait, et determiné par chromatographie gazeuse la teneur en acide céto- 9 . Pour cette dernière opération les mesures n'ont pas été effectuées selon la méthode classique, dans laquelle les acides sont transformés en leurs esters méthylés correspondants par addition de diazométhane. Elles ont été effectuées sur un chromatographe à gaz de la firme Hewlett-Packard de la série $5720 \mathrm{~A}$, équipé d'un FID et d'une colonne acier (longueur $1,8 \mathrm{~m}$; diamètre extérieur $3,2 \mathrm{~mm}$; intérieur $2,7 \mathrm{~mm} ; 2,5 \% \mathrm{SP}$ 1000; Chromosorb G; AW-DMCS; 80-100 mesh). La température dans le four des colonnes était maintenue constante, à $240^{\circ} \mathrm{C}$ (chambre d'injection $280^{\circ} \mathrm{C}$, détecteur $300^{\circ} \mathrm{C}$ ).

On a utilisé l'azote comme gaz vecteur (vitesse de débit $40 \mathrm{ml} / \mathrm{min}$ ).

Pour effectuer les mesures on a broyé la tête d'une ouvrière du Cap dans une coupelle Eppendorf et dilué avec 50-100 $\mu 1$ d'acétone. Après le maintien en chambre froide pendant 15 heures, on a effectué la mesure en chromatographie gazeuse en introduisant $1-3 \mu \mathrm{l}$ de l'extrait par acétone dans la chambre d'admission des échantillons. Si l'on traitait les têtes de plusieurs abeilles de même âge dans un même échantillon, la quantité de solution était élevée proportionnellement. 
Chez les abeilles du Cap provenant de la colonie avec reine, on a pris 10 abeilles par tranche d'âge. Sur les 5 premières abeilles on a effectué une mesure et sur les 5 suivantes une autre mesure. Par contre chez les ouvrières du Cap provenant des cagettes expérimentales on a procédé en majorité à des mesures individuelles. Tous les $2^{\mathrm{e}}, 3^{\mathrm{e}}, 5^{\mathrm{e}}, 7^{\mathrm{e}}, 8^{\mathrm{e}}$ et $11^{\mathrm{e}}$ jour de vie on a analysé de 2 à 6 têtes d'abeilles.

A l'aide d'une solution témoin d'acide céto- 9 synthétique dilué dans l'acétone, que l'on injectait après chaque chromatogramme d'extrait de tête, on a pu savoir si de l'acide céto-9 était présent dans l'échantillon analysé et, si oui, dans quelle proportion (Fig. 1).

L'évaluation quantitative a découlé de la détermination approximative de la surface des pics : hauteur d'un pic x base médiane (base médiane = largeur du pic à la moitié de la hauteur entre le maximum et la ligne de base).

Entre le $5^{\mathrm{e}}$ et le $7^{\mathrm{e}}$ jour d'expérimentation les abeilles des cagettes expérimentales ont commencé à construire des cellules sur le morceau de rayon, ce qui indique déjà que les ouvrières accompagnatrices acceptaient l'ouvrière du Cap comme "reine de substitution ".

Au $9^{\text {e }}$ jour de leur vie $79 \%$ des ouvrières du Cap avaient commencé à pondre.

Toutes les ouvrières du Cap provenant de la ruche avec reine ont montré des ovaires non développés et chez aucune des ouvrières testées on n'a pu mettre en évidence d'acide céto-9.

L'acide ceto- 9 était par contre présent chez $78 \%$ des ouvrières du Cap provenant des cagettes expérimentales. Il a été enregistré pour la première fois chez les ouvrières de 4 jours avec une moyenne de $12,5 \mu \mathrm{g} /$ ouvrière du Cap. La quantité d'acide céto-9 mesurée a augmenté ensuite continuellement et atteint chez les ouvrières de plus de 100 jours une moyenne de $412,9 \mu \mathrm{g} /$ abeille. Aucune diminution de la teneur en acide céto- 9 n'a été mise en évidence chez les ouvrières qui, au moment de la mesure, n’étaient pas pondeuses.

Le développement ovarien des ouvrières du Cap, semble navoir aucune influence sur la formation de l'acide céto-9, bien que la majorité des ouvrières du Cap, chez lesquelles on a mesuré la quantité d'acide céto- 9 , possédât des ovaires développés et fût en partie pondeuse.

\section{LITERATUR}

Anderson R. H., 1963. - The laying worker in the Cape honeybee, Apis mellifera capensis. J. Apic. Res., 2, 85-92.

Barbier M., Lederer E., 1960. - Structure chimique de la substance royale de la reine dabeille (Apis mellifica L.). C.R. Acad. Sci. Paris, 250, $4467-4469$.

Bertholf L. M., 1942. - Effect of certain biological factors on the longevity of caged bees. J. Econ. Entomol., 35, 887-891.

ButLer C. G., 1957 a. - The process of queen supersedure in colonies of honey-bees (Apis mellifera L.). Insectes sociaux, 4, 211-223.

BUTLER C. G., 1957 b. - The control of ovary development in worker honeybees (A. mellifera). Experientia, 13, 256.

Butler C. G., 1959. - Queen substance. Bee World, 40 (11), 269-275.

Butler C. G., Paton P. N., 1962. - Inhibition of queen rearing by queen honeybees (Apis mellifera L.) of different ages. Proc. R. ent. Soc. Lond. A, 37, 114-116.

CAllow R. K., Johnston N. C., 1960. - The chemical constitution and synthesis of queen substance of honeybees (Apis mellifera L.). Bee World, 41, 152.

DARCHEN R., 1957. - La reine d'Apis mellifica, les ouvrières pondeuses et les constructions cirières. Insectes sociaux, 4 (1), 322-325.

DARCHEN R., 1960. - Les régulations neurohormonales de l'instinct constructeur des ouvrières d'Apis mellifica. Ann. Abeille, 3 (4), 329-333. 
GaRY N. E., 1961. - Queen honey bee attractiveness as related to mandibular gland secretion. Science, $133,1479.1480$.

Groot A. P. de, vooge S., 1954. - On the ovary development in queenless worker bees (Apis mellifica L.). Experientia, i0, 384-385.

Hess G., 1942. - Über den Einfluss der Weisellosigkeit and des Fruchtbarkeitsvitamins E auf die Ovarien der Bienenarbeite1in. Beih. schweiz. Bienenzig, 1, Heft 2.

Hesse B., 1977. -- Ovarentwicklung und Eiablage bei Arbeiterinnen verschiedener Rassen von Apis mellifera (vergleichende Untersuchungen). Examensarbeit am Inst. f. Bienenkurde, Obersursel.

Mánkizio A., 1946. -- Beobachtungen über die Lebensdauer und den Futterverbrauch gefangen gehaltener Bienen. Beih. schweiz. Bienenzig, 2, Heft 13.

Mauruzio A., 1961. - Lebensajuer und Altern bei der Honigbiene (Apis mellifica L.). Geroniologie, $5_{3}$ 110-128.

Onions G. W., 1912. -- South African "Fertile - Worker Bees\%, Ayric. J. Un. S. Afi., 3 (5), $720-728$.

PAiv J. 1966. - Nouveau modèle de cagettes experimentales pour le maintion dabeiles con captivité. Ann. A beilie, 3, $71-76$.

Pain J., Barrier M., Boguanowshi D., Lederek E. 1962. - Chemistry and biological activity of the secretions of queen and worker honeybes (Apis melifical. Comp. Biochem. Fhysiol., 6, 233241.

Pall J., Barbier M., Roger 8., 196\% - Dosages individuels des acides céc-9 décene-2 oique et hydroxy 10 décène-2 oique dans les têtes des reines et des ouvrières d'abeilles. Ann. Abeille, 10, 45-52.

PAYN J. Roger B, 1976. - Vartation de la teneur en acide céto 9 décéne-2 vique en fonction de l'âge che $z$ les reines vienges dabeille (Apis mellifica liguitica S.). C.R. Acad. Sc. Paris, 283 (7), $797-799$.

PAIN J., Rogek B., 1978 ,- Rythme circadien des acides céto 9 décène 2 oique, phéromone de la reine et hydroxy-10 décène-2 oique des ouvières d'abeilles Apis ncllifica ligustica S. Apidologic, 9 (4), 263272.

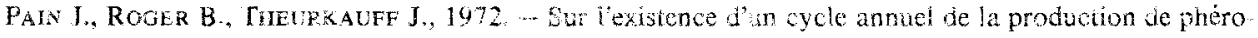

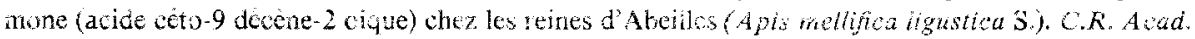
Sci. Paris, D, 275, 2399-2402.

Pam J., Roger B.. Theurkauff J., 1974, - Mise en éviderce d'un oyelu saisonnier de la tencur en acides céto-9 et hydroxy-9 décène 2 vique des têtes de reines vierges d'abeilic (Afis mollifica ligusticio S.). Apiäologie, 5 (4), 319-355.

Roger B., 1971. - L'influence de la rence d abcille (Aplis ncllifica L.) sur la prise de noun riture des ouvrieres accompagnatrices. Apidologic, 2 (2), 123-155.

Rotrner F., 1976. - The Cape bee - a biologlcal curiosity. Inturn. Symposiom on tiology, taxonomy and selection of African Bees, Pretoria 17. - 25.11.76.

RuTINer F., 1977, - The problem of the cape bee (Apis melifra cafensis Fscholtz) : parthenogentesissize of population - evolution. Apidologie, 8 (3) 28 i-294.

RutTNer F., Koeniger N., Veith H. J., 1976. - Queen substance bei cieriegunuen Arbeiterinnen der Honigbiene (Apis mellifica L.j. Nain wissentichaften, 63, 434.

SAKAGAmi S. F. AKHik Y Y. 1958. -- Comparison ol ovarian size and mumber of ovaricles between workers of the Japanese and European honeybees. Kontyu, 26, 103 109

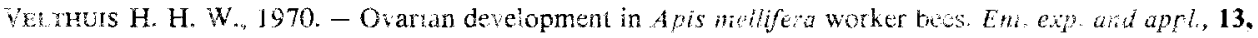
$377-394$.

Voogn S, 1455, - Inhitition of overy development w whe bees by tration ind of tho quen. Expe rieniia, 11, $181-182$.

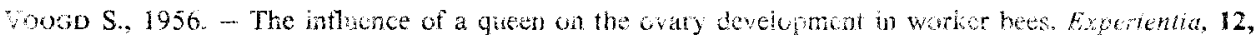
$199-201$. 